Bangladesh J. Sci. Res. 30(1\&2): 23-33, 2017 (December)

\title{
IMPORTANCE OF MATERNAL EDUCATION ON ANTENATAL CARE VISITS IN BANGLADESH
}

\author{
Khandoker Akib Mohammad*, Fatima-Tuz-Zahura and Md. Morshadur Rahman \\ Department of Statistics, University of Dhaka, Dhaka-1000, Bangladesh
}

\begin{abstract}
An attempt has been made to examine whether maternal education influences the antenatal care (ANC) visit in Bangladesh using sequential logistic regression models with an interaction between maternal education and place of residence. For the purpose of analysis, Bangladesh Demographic and Health Survey (BDHS), 2014 data set have been used. The findings emerged from the study show a significant increase of adequate ANC visits among pregnant women with the increase in maternal education level. Moreover, interaction between maternal education and place of residence provides a significant effect on complete ANC visits. The finding justifies an influential impact of maternal education on ANC visits over place of residence. Female participation in the education programs needs to be increased because maternal education signifies a strong positive association with ANC visits.
\end{abstract}

Key words: BDHS, Antenatal care, Logistic regression model, Odds ratio

\section{Introduction}

In last few decades, maternal morbidity and mortality have been reduced significantly in developed countries (Donnay 2000). However, the situation is different in many developing regions of the world. In order to ensure better health of pregnant women and delivery of a healthy baby as well, it is necessary to have at least four antenatal care visits (ANC) for uncomplicated pregnancies and more are necessary only in cases of complications shown by empirical evidence (Villar et al. 2001). According to a worldwide survey study, maternal education is found to play a vital role on maternal mortality. Moreover, maternal mortality rate tends to be higher in countries where female literacy rate is lower than their male counterparts (McAlister and Baskett 2006). This is because mother's health care seeking behavior is highly influenced by her education and mothers with higher education use health care services to a greater extent than the mothers with lower education. Education makes women aware of the effects of poor health and makes them understand the demand and utilization of health care (Rahman et al. 2008). The effect of distance to health care centers is minimized by educated mothers as their own health and child health are their first priorities (Matsuyama 2012). Several other studies support that the utilization of ANC is influenced by women's autonomy and decision-making power over their own health (Matsumura and Gubhaju 2001, Raghupathy 1996). The place of residence, urban or rural area, is also a factor affecting the use of ANC. Some studies have shown differences between the urban and rural areas in knowledge, attitude and practices of women towards antenatal care (Kishk 2002, Alexandre

*Author for correspondence: <akibfarhan444@yahoo.com>. 
et al. 2005). Moreover, the urban-rural differential in ANC coverage continues to be large in Bangladesh. Almost $74 \%$ of urban women received ANC from a medically-trained provider compared with $49 \%$ of rural women (BDHS 2011).

The Health, Population and Nutrition Sector Development Program (HPNSDP) results framework sets a target of 50 per cent of pregnant women making at least four antenatal care visits to be achieved by 2016. Though in Bangladesh, the percentage of pregnant women who made four or more ANC visits has increased, from 17 per cent in 2004 to the current level of 31 per cent, this is far behind in reaching the target (BDHS 2014). Therefore, the study of ANC visits has earned great attention to the researchers and policy makers. In a few studies, it was found that factors such as mother's age at birth, gender of child, wealth index, birth order, exposed to media etc. may play vital role in influencing ANC visit during pregnancy (Magadi et al. 2000, Nisar and White 2003, Overbosch et al. 2004, Sibley et al. 2004, Sharma 2004, Alam et al. 2005, Kabir et al. 2005, Shahjahan et al. 2012, Rahman et al. 2017). An attempt has been made to identify whether maternal education influences ANC visit during pregnancy in Bangladesh using sequential logistic regression models. It is also examined the relative importance of maternal education and place of residence by incorporating the interaction effect. For the purpose of analysis, Bangladesh Demographic and Health Survey (BDHS), 2014 data set have been used.

\section{Materials and Methods}

The study has utilized the nationwide data of BDHS 2014 (individual recode data). A two stage stratified sampling procedure was used to obtain the data, where in the first stage, 600 clusters were selected from both the rural (393 clusters) urban areas (207 clusters) of the country and in the second stage of sampling, a systematic sample of 30 households was selected on average from each enumeration area. In the 2014 BDHS, a total of 17863 ever married women of age 12-49 from the selected households were interviewed who had given birth in the three years preceding the survey and they were asked a number of questions about maternal and child health care. Information on ANC was assessed for women who gave birth in the three years preceding the survey. Among women with two or more live births during the three-year-period, data refer to the most recent live birth only.

The main aim of this paper is to identify the influence of maternal education on ANC visit during pregnancy in Bangladesh. In the analysis, to collect the data on ANC visit during pregnancy, we have considered last birth of women who gave birth in the three years preceding the survey and finally a total of 4470 women were selected in this study.

On the basis of literature review on ANC visit during pregnancy, (Pamela et al. 2005, Simkhada et al. 2006, Simkhada et al. 2007, Islam and Odland 2011, Shahjahan et al. 2012, Christopher et al. 2013, Edward et al. 2013, Anchang-Kimbi et al. 2014) the factors considered in this paper are age of mother's at birth of index children in years $(<20,20-30,>30)$, mother's education level (no, primary, secondary, higher), place of residence (urban, rural), birth order 
number (first birth, second and third birth, and others), wealth index (poor, middle, rich), exposure to media (yes, no), mother's working status (yes, no) and aware of community clinic (yes, no).

In studies with a dichotomous dependent variable, a logistic regression model is a common choice, which estimates the effects of a set of explanatory variables on the dependent variable. In this study, the dependent variable, ANC visits during pregnancy has been coded as a dichotomous variable where ANC visit $=1$ for the complete ANC visits (ANC visits $\geq 4$ ) and 0 for fewer ANC visits(ANC visits $\leq 3$ ). Hence, to model the relationship of maternal education and ANC visit, a sequential logistic regression modeling technique has been applied controlling the effects of other demographic and socio-economic variables.

To examine the association between maternal education and antenatal care visits, sampling weights have been used. As this paper aims to determine the influence of maternal education on antenatal care visits, not to find any national level estimates, the design weights have not been considered in the regression analysis (Winship and Radbil 1994). For the purpose of computation, STATA 12.1 has been used.

\section{Results and Discussion}

Three major steps have been performed in this study for the purpose of analysis. Percentage distribution of each category of the selected variables has been given to show the pattern of the data on selected factors. Then weighted Chi-square test is performed to identify the unadjusted potential factors associated with ANC visits during pregnancy. Finally, stepwise logistic regression models have been fitted to the data to find out the effect of maternal education on ANC visits controlling the effects of other important demographic and socio-economic variables.

Univariate analysis: It is observed that more than half of the mothers $(73.10 \%)$ gave their birth at their young age (age less than 20 years), while $26.40 \%$ of mothers gave birth when they were in age group 20 - 30 and $0.50 \%$ of mother's age at birth was more than 30 years. Among all women, $14.20 \%$ have no education while 28 per cent completed primary and 47.70 per cent completed their secondary education. Moreover, there are a few women (10.10\%) who completed their higher education. The distribution of respondents with respect to place of residence is 73.90 and $26.10 \%$ for the rural and urban areas, respectively. It is also observed that $39.90 \%$ of the children are the first babies of their mother. Highest number of children were from poor families $(40.60 \%)$ whereas $19.10 \%$ children were from middle class families. It is seen that a large number of mothers $(61.70 \%)$ were found to be exposed to media. Among all women, only $23.50 \%$ are working. Moreover, it can be observed that only $28.50 \%$ of women are aware of community clinic.

In this paper, the event of interest is the complete ANC visits during pregnancy. Out of 4470 observations, only $31.30 \%$ women experienced the event. 
Bivariate analysis: An attempt has been made to find out the unadjusted association of selected factors with ANC visit during pregnancy. The cross tabulation with Chi-square test is used to determine whether a specific covariate has significant association with ANC visits during pregnancy. The results are given in Table 1.

Table 1. Percentage distribution of ANC visits by selected covariates with Chi-square test p-value.

\begin{tabular}{|c|c|c|c|c|}
\hline \multirow{2}{*}{$\begin{array}{l}\text { Covariates } \\
\text { Total }\end{array}$} & \multirow{2}{*}{$\begin{array}{c}\text { Sample size } \\
4470\end{array}$} & \multicolumn{2}{|c|}{ ANC visits } & \multirow{2}{*}{$\begin{array}{c}\text { Chi-square test } \\
\text { (p-value) }\end{array}$} \\
\hline & & Visits $\leq 3$ & Visits $\geq 4$ & \\
\hline \multicolumn{5}{|c|}{ Mother's age at birth } \\
\hline Below 20 & 3241 & 72.60 & 27.40 & \\
\hline $20-30$ & 1208 & 58.40 & 41.60 & $<0.001$ \\
\hline Above 30 & 21 & 50.00 & 50.00 & \\
\hline \multicolumn{5}{|c|}{ Maternal education } \\
\hline No & 604 & 85.90 & 14.10 & \\
\hline Primary & 1228 & 79.30 & 20.70 & $<0.001$ \\
\hline Secondary & 2120 & 63.30 & 36.70 & \\
\hline Higher & 518 & 41.10 & 58.90 & \\
\hline \multicolumn{5}{|l|}{ Residence } \\
\hline Rural & 3026 & 73.80 & 26.20 & $<0.001$ \\
\hline Urban & 1444 & 54.30 & 45.70 & \\
\hline \multicolumn{5}{|l|}{ Birth order } \\
\hline 1 st & 1817 & 63.10 & 36.90 & \\
\hline 2nd and 3rd & 2030 & 68.90 & 31.10 & $<0.001$ \\
\hline 4 th + & 623 & 84.30 & 15.70 & \\
\hline \multicolumn{5}{|c|}{ Wealth index } \\
\hline Poor & 1783 & 82.40 & 17.60 & \\
\hline Middle & 856 & 72.70 & 27.30 & $<0.001$ \\
\hline Rich & 1831 & 53.10 & 46.90 & \\
\hline \multicolumn{5}{|c|}{ Exposed to media } \\
\hline Exposure & 2775 & 59.80 & 40.20 & $<0.001$ \\
\hline Unexposure & 1695 & 83.00 & 17.00 & \\
\hline \multicolumn{5}{|c|}{ Working status } \\
\hline Yes & 976 & 72.20 & 27.80 & 0.003 \\
\hline No & 3494 & 67.70 & 32.30 & \\
\hline \multicolumn{5}{|c|}{ Aware of community clinic } \\
\hline Yes & 1353 & 70.10 & 29.90 & 0.193 \\
\hline No & 3117 & 68.20 & 31.80 & \\
\hline
\end{tabular}

It is observed that complete ANC visits were highest for children whose mother's age at birth were above 30 and lowest for children whose mother's age at birth were below 20. Mother's level 
of education has a similar directional relationship with complete ANC visit. For example, complete ANC visit is highest among the children whose mothers are higher educated. Similar results also exist among the categories of wealth index; as wealth increases, the extent of complete ANC visit among women increases. It can be observed that complete ANC visit is higher in urban area compared to rural area. Complete ANC visit is higher for the children who were the first babies of their parents. Moreover, complete ANC visit is higher for the children whose mothers were exposed to media. However, children whose mothers were working outside their home, have lower complete ANC visits.

It is clear from Table 1 that all the selected covariates except community clinic awareness were found to have significant association with ANC visits during pregnancy as p-values were less than 0.05 .

Logistic regression models: The current paper aims to focus the influence of maternal education on complete ANC visit $(\geq 4)$. For the purpose of analysis, stepwise logistic modeling technique has been employed. In Model 1, only maternal education was considered as covariate. In Model 2, some demographic variables have been incorporated along with covariate considered in Model 1 to examine how demographic factors change the influence of maternal education on complete ANC visits. Similarly, the socioeconomic variables were added to construct Model 3. Finally, Model 4 involves interaction component between maternal education and place of residence along with covariates considered in Model 3. Estimated regression coefficient, odds ratio along with p-value for all models are shown in Table 2.

From Model 1, it is clear that there is a highly significant unadjusted association between maternal education and complete ANC visits. Odds ratio reported in Table 2 confirms that illiterate women have $90.1 \%$ lower odds of having at least 4 ANC visits compared to higher educated women. Moreover, mothers with primary and secondary education have, respectively 84.0 and $66.4 \%$ lower odds of having complete ANC visits compared to mothers with higher education.

In Model 2, considering demographic variables (mother's age at birth and birth order) and maternal education as covariate, we observed that mothers with education level primary, secondary and no education have, respectively 79.8, 60.1 and $86.6 \%$ lower odds of occurring complete ANC visits compared to higher educated mothers and these findings are highly significant. However, a significant difference of receiving complete ANC visits has been observed between categories of mother's age at birth. Women who were below 20 years at the time of birth are 0.421 times likely to receive at least four ANC visits compared to the women who were above 30 years at the time of their first birth. Moreover, mothers during their first pregnancy have 55.8\% higher odd of having complete ANC visits compared to pregnancies of higher order (4th and above). In addition, $46.8 \%$ higher odd of having complete ANC have been found among mothers during their second and third pregnancies compared to their pregnancies with order 4th and above. 


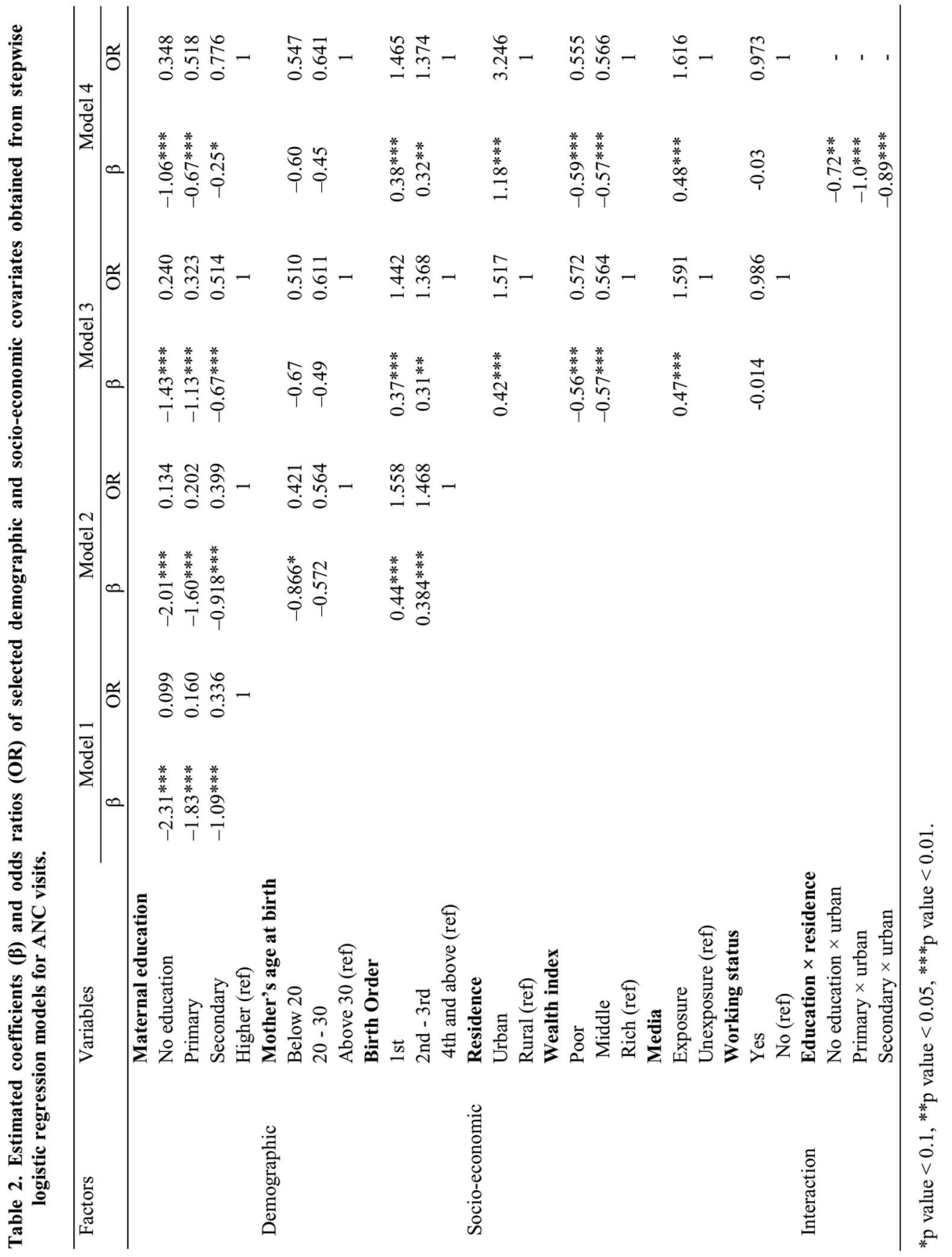


Result obtained from Model 3 confirms that maternal education provides a significant association with complete ANC visits controlling some important socio-economic and demographic variables. Result shows that women who have no education, primary and secondary education, respectively have $0.240,0.323$ and 0.514 times odd to have complete ANC visits compared to the women having higher education. In addition, higher birth order is inversely and significantly associated with the number of ANC visits by expecting mothers. It is seen that for the first baby and babies with order 2nd and 3rd, women have, respectively 44.2 and $36.8 \%$ higher odd to have at least four ANC visits compared to the other babies. It is observed that women from urban area have $51.7 \%$ higher odd of having complete ANC visits compared to the women from rural area and it is proved to be an influential factor for ANC visits at $1 \%$ level of significance. Moreover, women from poor $(\mathrm{OR}=0.572)$ and middle $(\mathrm{OR}=0.564)$ families are significantly less likely to experience complete ANC visits compared to the women from rich families. Also, it can be observed that the odd of having complete ANC visits for the women who are exposed to media is $59.1 \%$ higher compared to the women who are not exposed to media and is a potential factor for ANC visits at $1 \%$ level of significance. The variables mother's age at birth and working status are found insignificant in Model 3.

The final model, Model 4, incorporates interaction terms between mother's education and residence along with the previously included socioeconomic and demographic variables. Under this model it is observed that women who have no education, primary and secondary education, have respectively $65.2 \%$ (p-value $<0.01$ ), 48.2\% (p-value $<0.01$ ) and $22.4 \%$ (p-value $<0.1$ ) lower odd to have complete ANC visits compared to the women having higher education. The variable birth order is also found to have significant effect on complete ANC visits by expecting mothers. It is seen that for the first baby and babies with order 2nd and 3rd, women have respectively 46.5 and $37.4 \%$ higher odd to have at least four ANC visits compared to the babies with order 4 and above. It is observed that women from urban area have 3.246 times odd of having complete ANC visits compared to the women from rural area and it is found to be a significant factor for ANC visit at $1 \%$ level of significance. Moreover, women from poor $(\mathrm{OR}=0.555)$ and middle $(\mathrm{OR}=0.566)$ families are significantly less likely to experience complete ANC visits compared to the women from rich families. Also, it can be observed that the odd of having complete ANC visits for the women who are exposed to media is $61.6 \%$ higher compared to the women who are not exposed to media and the result is found significant at $1 \%$ level of significance.

Table 3 shows the odds ratios for interactions that have been calculated according to the work done by Chen (2003). The interaction between mother's education and residence reveals that women residing in rural areas with education level secondary, primary and illiterate have 22.4, 48.2 and $67.5 \%$ lower odd of having complete ANC visits compared to higher educated women who live in rural areas. Moreover, an illiterate woman who lives in urban area is $45.1 \%$ less likely to have at least four ANC visits than a woman with higher education who lives in rural area. It is also observed that the odd of having complete ANC visits for women who attended primary 
school and live in urban areas are $38.7 \%$ lower compared to the women who have higher education and live in rural areas. However, women from urban areas with secondary education are 1.04 times as likely as to have complete ANC visits than women from rural areas with higher education.

Table 3. Estimated odds ratios of interaction between maternal education and place of residence.

\begin{tabular}{ll}
\hline Interactions & Odds ratio \\
\hline No education $\times$ urban & 0.549 \\
Primary $\times$ urban & 0.613 \\
Secondary $\times$ urban & 1.04 \\
Higher $\times$ urban & 3.246 \\
No education $\times$ rural & 0.328 \\
Primary $\times$ rural & 0.518 \\
Secondary $\times$ rural & 0.776 \\
Higher $\times$ rural & 1 \\
\hline
\end{tabular}

Maternal education is one of the key factors that enhance knowledge among mothers regarding maternal and child health during pregnancy period which is a key indicator of safe and healthy delivery. In this study, the importance of maternal education has been conducted on ANC visits in Bangladesh using sequential logistic regression models. The first model provides unadjusted effect of maternal education on ANC visits. Models 2 and 3 include some important demographic and socio-economic variables, respectively that were found significant in chi-square test. Finally Model 4 involves both demographic and socioeconomic variables along with interaction term between maternal education and place of residence. The Model 1 shows a significant unadjusted association between maternal education and ANC visits. After controlling the effect of demographic and socio-economic variables in Models 2, 3 and 4, it is found that though the magnitude of the effect of maternal education on ANC visits is decreasing but still it remains significant on ANC visits. The findings emerged from the study show a significant increase of adequate ANC visits among pregnant women with the increase in maternal education level. Moreover, interaction between maternal education and place of residence provides a significant effect on complete ANC visits. A higher chance of having complete ANC visits has been found significant among mothers with secondary education residing in urban area compared to higher educated mothers who belong to rural area. According to this finding it can be observed that the difference of the mother's education level from secondary to higher is getting dominated by place of residence. In the case of illiterate and primary educated mothers, the interactions reveal a noticeable result. Despite living in urban areas, illiterate and primary educated mothers have lower odds of receiving adequate ANC visits compared to mothers residing in rural areas who have higher education. This finding justifies an influential impact of maternal education on 
ANC visits over place of residence. This may happen because improved educational status of mothers may help them to enrich their knowledge to demand and seek proper health care important to negate pregnancy complications.

Though Bangladesh has witnessed a huge improvement in ANC visits during the last decade, still the study of ANC visit has become one of the most important issues because of lower rate compared to other developed and developing countries. Findings of the study prove maternal education as an important factor for adequate ANC visits. Therefore, emphasis should be given more on educating women as well as improving maternal health care services. Government efforts should be designed to enhance female education at least secondary level for future favorable health outcomes. Moreover information, education and communication on ANC must be intensified to reach the rural mothers which consequently alleviate maternal mortality rate and promote better maternal and child health.

\section{Acknowledgement}

The authors would like to thank the authority of National Institute of Population Research and Training (NIPORT), Bangladesh for allowing them to use the BDHS, 2014 data for their analysis. Moreover, the authors would like to thank Professor Dr. Wasimul Bari, Department of Statistics, University of Dhaka for his help and valuable comments on the preparation of this manuscript.

\section{References}

Alam, AY., AA. Qureshi, MM. Adiland H. Ali. 2005. Comparativestudy of knowledge, attitude and practices among antenatal care facilities utilizing and non-utilizing women. Journal of the Pakistan Medical Association 55(2): 53-56.

Alexandre PK., G. Saint-Jean,L. Crandall and E. Fervin. 2005.Prenatal care utilization in rural areas and urban areas of Haiti. Rev. Panam Salud Publica.18(2): 84-92.

Anchang-Kimbi, KJ., AE. Achidi, OT. Apinjoh, NR. Mugri, FH. Chi, BR. Tata, B. Nkegoum, N. MJ. Mendimi, E. Sverremark-Ekström and M. Troye-Blomberg. 2014. Antenatal care visit attendance, intermittent preventive treatment during pregnancy (IPTp) and malaria parasitaemia at delivery. Malaria Journal 13: 162.

Bangladesh Demographic and Health Survey (BDHS). 2011. NIPORT, Dhaka, Bangladesh; Mitra and Associates, Dhaka, Bangladesh.

Bangladesh Demographic and Health Survey (BDHS). 2014. NIPORT, Dhaka, Bangladesh; Mitra and Associates, Dhaka, Bangladesh.

Chen, J.J. 2003. Communicating Complex Information: The Interpretation of Statistical Interaction in Multiple Logistic regression Analysis. American Journal of Public Health 93(9): 1376-1377.

Christopher, P., M. Arantza, W. Florence, AA. Nana, C. Samuel, M. Lucinda, JH. Mary, H. Abraham, T. Harry, K. Linda, O. Peter and P. Robert 2013. Factors Affecting Antenatal Care Attendance: Results from Qualitative Studies in Ghana, Kenya and Malawi. PLOS ONE 8(1).

Donnay, F. 2000. Maternal survival in developing countries: what has been done, what can be achieved in the next decade. Int. J. Gynaecol. Obstet. 70(1): 89-97. 
Edward, NA., S. Bernardin and A. Eric. 2013. Determinants of utilization ofantenatal care services in developing countries, Recent evidence from Ghana. African Journal of Economic and Management Studies 4(1): 58-73.

Islam, M. R. and J. O. Odland. 2011. Determinants of antenatal and postnatal care visits among Indigenous people in Bangladesh: a study of the Mru Community. Rural Remote Health 11(2): 1672.

Kabir, M., Z. Iliyasu, IS. Abubakar and AA. Sani 2005. Determinant of utilization of antenatal care services in Kumbotso village, Northern Nigeria. Tropical Doctor 35: 110-111.

Kishk, N.A. 2002. Knowledge, attitudes and practices of women towards antenatal care: rural-urban comparison. J Egypt Public Health Assoc. 77(5-6): 479-498.

Magadi, M.A., N.J. Madise and R.N. Rodrigues 2000. Frequency and timing of antenatal care in Kenya: explaining the variations between women of different communities. Social Science and Medicine 51(4): 551-561.

Matsuyama, A. 2012. Effects of women's education on antenatal care seeking behavior in Nepal: Qualitative and Quantitative Approaches. Center of International Collaborative Research and Institute of Tropical Medicine, Nagasaki University.

Matsumura, M. and B. Gubhaju 2001. Women's status household structure and the utilisation of maternal health services in Nepal. Asia-Pacific Population Journal 16(1): 23-44.

McAlister, C. and T.F. Baskett 2006. Female education and maternal mortality: a worldwide survey. $J$. Obstet. Gynaecol. Can. 28(11):983-90.

Rahman, M.M., M.R. Islam and A.Z. Islam 2008. Rural-urban differentials of utilization of antenatal health care services in Bangladesh. Health Policy and Development 6(3): 117-125.

Nisar, N. and F. White 2003.Factors affecting utilization of antenatal care among reproductive age group women (15-49 years) in anurbansquatter settlement of Karachi. Journal of the Pakistan Medical Association 53(2):47-53.

Overbosch, G.B., N.N.N. Nsowah-Nuamah, G.J.M. van den Boom and L. Damnyag. 2004. Determinants of antenatal care use in Ghana. Journal of African Economies 13(2): 277-301.

Pamela, L., P. Janis, W. Trecia, C. Sarnia, W. Maynard and P. Teuila 2005. Factors affecting antenatal care attendance by mothers of Pacific infants living in New Zealand. The New Zealand Medical Journal 118: $1-10$.

Raghupathy, S. 1996. Education and the use of maternal health care in Thailand. Social and Medicine 43(4): 459-471.

Rahman, A., MK. Nisha,, T. Begum, S. Ahmed, N. Alam and I. Anwar. 2017. Trends, determinants and inequities of 4+ ANC utilisation in Bangladesh. Journal of Health, Population and Nutrition 36(1):2.

Shahjahan, M., AH.Chowdhury, J. Akter, A. Afroz, MM. Rahman and AM. Hafez 2012.Factors associated with use of antenatal care services in a rural area of Bangladesh. South East Asia Journal of Public Health 2(2): 61-66.

Sharma, B., 2004. Utilisation of antenatal care services in Nepal. Nepal Population Journal 11(10): 79-97.

Sibley, LM., TA. Sipeand and M. Koblinsky 2004. Does traditional birth attendant training increase use of antenatal care? A review of the evidence. Journal of Midwifery \& Women's health 49(4): 298-305.

Simkhada, BD., ER. Van Teijlingen, M. Porter and P. Simkhada 2006. Major problems and key issues in maternal health in Nepal. Kathmandu University Medical Journal 4(2): 258-263. 
Simkhada, B., ER. Van Teijlingen, M. Porter and P. Simkhada. 2007. Factors affecting the utilization of antenatal care in developing countries: systematic review of the literature. Journal of Advanced Nursing Research 61(3): 244-260.

Villar, J. and P. Bergsj. 2002. WHO antenatal care randomized trial: Manual for the implementation of the new model. Geneva. World Health Organ.

Winship, C. and L. Radbil 1994. Sampling weights and regression analysis. Sociological Methods and Research 23(2): 230-257.

(Manuscript received on 5 April, 2017; revised on 6 June, 2017) 